\title{
IMPACTO DA VÍDEOCIRURGIA NA PREVENÇÃO DE ADERÊNCIAS
}

\author{
SERGIO EDUARDO ALONSO ARAÚJO' ${ }^{1}$, PEDRO PAULO DE PARIS CARAVATTO ${ }^{1}$, ALEXANDRE JIN BOK AUDI \\ CHANG $^{1}$, FÁBIO GUILHERME C. M. DE CAMPOS ${ }^{1}$, MANOELA SOUSA $^{1}$
}

\author{
${ }^{1}$ Disciplina de Coloproctologia do Departamento de Gastroenterologia do Hospital das Clínicas da Faculdade de \\ Medicina da Universidade de São Paulo
}

ARAUJO SEA, CARAVATTO PPP, CHANG AJBA, CAMPOS FGCM, SOUSA M. Impacto da Vídeo-Cirurgia na Prevenção de Aderências. Rev bras Coloproct, 2006;26(2):208-216

RESUMO: Uma das vantagens aventadas da vídeo-cirurgia é a possibilidade de formar menos aderências pós-operatórias. As evidências deste efeito resultam de trabalhos clínicos e experimentais, mas o real impacto desta via de acesso neste sentido ainda não foi comprovado. $O$ objetivo da presente revisão foi avaliar as evidências científicas disponíveis sobre o assunto. MATERIAL E MÉTODOS: revisão da literatura pertinente. RESULTADOS: As aderências pós-operatórias foram analisadas no sítio da operação e nas incisões praticadas, porém existem poucas informações sobre aderências em locais não operados. Aderências pós-operatórias são menos freqüentes ou intensas quando se considera a via de acesso por vídeo. A despeito deste dado experimental, os desfechos clínicos de menor dor pélvica, menor número de admissões ou reoperações por obstrução intestinal e menor ocorrência de infertilidade ainda não podem ser claramente atribuídos a esta via de acesso, especialmente quando se consideram as cirurgias laparoscópicas avançadas, uma vez que nesta situação existe equivalência de área cruenta nas duas vias de acesso, à exceção da área associada às incisões. CONCLUSÕES: A via de acesso por vídeo está associada a menor formação de aderências, mas não protege de complicações relacionadas à sua ocorrência. Técnica operatória adequada e o uso de barreiras provavelmente estão mais fortemente associadas à menor formação de aderências do que a via de acesso aberta empregada para a realização das operações abdominais e pélvicas.

Descritores: Aderências, Laparoscopia, Obstrução Intestinal, Dor Pélvica, Infertilidade

\section{INTRODUÇÃO}

Aderências são conexões patológicas entre superfícies dentro das cavidades do corpo. Estas conexões ou pontes podem ser constituídas por fina lâmina de tecido conjuntivo, por lâmina tecidual mais espessa contendo vasos sanguíneos e nervos ou representar um contato direto entre a superfície de dois órgãos. Podem ser encontradas nas cavidades peritonial, pericárdica, pleural, uterina, no interior de articulações ou das câmaras oculares. Na cavidade abdominal são conhecidas como aderências peritoniais, uma vez que o peritônio está sempre envolvido. Sua incidência após operações é elevada, podendo ocorrer em até $93 \%$ das laparotomias. ${ }^{1}$

Aderências intraperitoniais podem ser benéficas, promovendo revascularização de superfícies isquêmicas como anastomoses intestinais. Entretanto,

Trabalho realizado na Disciplina de Coloproctologia do Departamento de Gastroenterologia do Hospital das Clínicas da Faculdade de Medicina da Universidade de São Paulo

Recebido em 17/05/2006

Aceito para publicação em 04/06/2006 
podem determinar obstrução intestinal e ser causa de aproximadamente $70 \%$ das reinternações por obstrução de intestino delgado ${ }^{2}$. São responsáveis por $15-20 \%$ dos casos de infertilidade secundária em mulheres e estão associadas a dor crônica abdominal e pélvica ${ }^{3,4}$. Além disso, prolongam o tempo operatório em relaparotomias, levando a enterotomias inadvertidas em aproximadamente $20 \%$ dos casos, o que acarreta maior incidência de complicações pós-operatórias, necessidade de cuidados intensivos e internação hospitalar prolongada ${ }^{5}$.

As operações abdominais representam a maior causa conhecida de formação de aderências, embora elas ocorram em até $10 \%$ de pacientes nunca submetidos a operações abdominais prévias ${ }^{6}$. Entendese que a vídeo-cirurgia possa levar a menor formação de aderências quando comparada à via convencional, como resultado de menores incisões, manipulação tecidual e sangramento.

O objetivo desta revisão foi avaliar criticamente as evidências científicas disponíveis acerca do papel da vídeo-cirurgia na formação de aderências e na prevenção de suas complicações pósoperatórias mais freqüentes.

\section{FISIOPATOLOGIA DA FORMAÇÃO DE ADERÊNCIAS}

Apesar de intuitiva, a fisiopatologia da formação de aderências é complexa, o que pode em parte explicar o porquê de sua relativa imprevisibilidade no que se refere ao local onde se formarão ou com qual intensidade. Sua formação se inicia a partir de uma resposta do peritônio a diferentes agressões, como infecção, irritação química e trauma direto, e inclui os sistemas da coagulação e fibrinolítico. ${ }^{7}$

A cavidade abdominal é revestida pelo peritônio, que consiste em uma camada única de células mesoteliais sustentada por uma membrana basal e uma camada subjacente de tecido conjuntivo. O trauma peritonial resulta em dano mesotelial, desencadeando uma resposta inflamatória local. As células mesoteliais se desprendem da membrana basal, criando áreas desnudas, desencadeando a produção de um amplo espectro de proteínas biologicamente ativas e de exsudato rico em proteínas. $\mathrm{O}$ exsudato peritonial contém altas concentrações de fibrinogênio.
A cascata de coagulação é ativada na cavidade peritonial, resultando na formação de trombina que ativa a conversão de fibrinogênio em fibrina. Devido à ativação do sistema fibrinolítico, qualquer depósito intra-abdominal de fibrina pode sofrer lise. Entretanto, após cirurgia abdominal e infecção, o equilíbrio entre coagulação e fibrinólise é afetado em favor do sistema de coagulação ${ }^{8-17}$. Desse modo, a fibrina forma depósitos que servirão de matriz para proliferação de tecido fibro-colagenoso e, portanto, levando à formação de aderências.

\section{CONSEQÜÊNCIAS DA FORMAÇÃO DE ADERENCIAS}

As principais conseqüências da formação de aderências são infertilidade, obstrução intestinal, dor pélvica crônica e o maior risco associado às reoperações (perfuração de vísceras ocas e de vasos intra-abdominais) ${ }^{18-21}$. A torção tubária, a estenose do suprimento ovariano e aderências intra-uterinas estão entre as maiores consequiências das aderências e estimase que estas podem representar causa da infertilidade em até $40 \%$ dos casos ${ }^{22}$.

Em estudo prospectivo envolvendo 224 pacientes que sofriam de dor abdominal crônica revelou-se que $82 \%$ possuíam apenas aderências e nenhuma outra doença associada. Estes pacientes foram submetidos a lise de aderências por laparoscopia. Após três meses, $74 \%$ dos pacientes estavam sem dor ou apresentaram melhora da dor ${ }^{23}$.

Aderências intraperitoniais representam a causa mais importante de obstrução intestinal, sendo responsáveis por $40 \%$ de todos os casos ${ }^{24,25,26}$ e até $70 \%$ dos casos de obstrução de intestino delgado ${ }^{27}$. Estudo publicado pelo Surgical and Clinical Adhesions Research (SCAR) Group ${ }^{28}$ envolvendo pacientes submetidos à cirurgia colorretal aberta demonstrou que a necessidade de reinternação atinge $9 \%$ no primeiro ano após a cirurgia abdominal $(2,1 \%$ como causa diretamente relacionada e $6,9 \%$ como causa provavelmente relacionada às aderências). As taxas de reoperação relacionadas às aderências após laparotomia variam de $1 \%$ até $2,6 \%^{18,29}$.

Estes resultados traduzem significativa morbidade associada às numerosas operações 
abdominais realizadas por via convencional. Ressaltese uma das conclusões do estudo SCAR, em que a grande maioria das reinternações por obstrução intestinal não é conduzida pelo cirurgião responsável pela operação que originou as aderências, o que gera uma subestimativa coletiva da intensidade do problema e, como resultado, maior dificuldade de se entender a necessidade de encontrar formas eficazes de evitá-lo.

\section{PREVENÇÃO DA FORMAÇÃO DE ADERÊNCIAS}

As duas maiores estratégias na prevenção ou redução da formação de aderências são representadas por ajustes na técnica cirúrgica e pelo uso de adjuvantes.

As modificações na técnica operatória fazem parte do treinamento do bom cirurgião e dela fazem parte todas as medidas utilizadas com a finalidade de reduzir o trauma cirúrgico, ou seja: controlar ou evitar a introdução de material estranho (talco de luvas cirúrgicas, por exemplo), prática de hemostasia cuidadosa, redução da exposição e ressecamento da superfície peritoneal com o emprego de compressas úmidas, uso de incisões adequadas, emprego de material cirúrgico delicado, cuidado no afastamento e manipulação das estruturas, controle e diminuição da contaminação.

Em 1886, Müller sugeriu o uso de solução salina para prevenir a ocorrência de aderências ${ }^{30}$. Desde então, inúmeros agentes foram investigados para a prevenção de aderências intra-abdominais. Eles são baseados na ativação da fibrinólise, alteração da coagulação, diminuição da resposta inflamatória, inibição da síntese de colágeno ou criação de uma barreira entre superfícies de feridas adjacentes.

A ativação do sistema fibrinolítico é considerada benéfica na prevenção de aderências intraabdominais. No final do século 19 preconizava-se o uso de agentes com capacidade fibrinolítica (tais como tiosinamina presente em bebida alcoólica), salicilato de sódio ${ }^{31} \mathrm{e}$ fósforo oral ${ }^{32}$. A estreptoquinase $\mathrm{e}$ estreptodornase foram os primeiros agentes com propriedades fibrinolíticas comprovadas que se mostraram eficazes em prevenir a formação de aderências em modelos experimentais ${ }^{33,34}$, embora sua eficácia nunca tenha sido comprovada em humanos. $\mathrm{O}$ fator ativador de plasminogênio tecidual (tPA) foi também estudado neste sentido. Embora tenha se provado a eficácia do tPA ${ }^{35-45}$, o risco de hemorragia tem sido o principal obstáculo para seu uso rotineiro. Anticoagulantes como heparina também são efetivos na prevenção de aderências ${ }^{46-55}$, embora o uso local de heparina em cirurgia abdominal permanece controverso devido ao risco de hemorragia.

Drogas antiinflamatórias foram testadas na prevenção de aderências, incluindo corticosteróides e inibidores da síntese de prostaglandinas ${ }^{56-65}$. Swolin ${ }^{57}$ obteve êxito em pacientes em que aplicou esteróides intraperitoniais, mas outros autores reportaram efeitos divergentes e, em alguns casos, deletérios ${ }^{58,59}$, associados intuitivamente à diminuição de resposta imune atribuídas a estas drogas.

A alteração do equilíbrio entre síntese e degradação de fibrina leva à persistência de aderências fibrinosas, que serão preenchidas por fibroblastos com deposição subseqüente de colágeno, resultando na formação de aderências fibrosas permanentes. Nagler e cols. ${ }^{66,67}$ demonstraram que o tratamento com halofuginona (um inibidor da síntese de colágeno tipo I) diminui a formação de aderências cirúrgicas induzidas experimentalmente. Testes clínicos ainda são aguardados.

A criação de uma barreira mecânica entre as superfícies das feridas é outro método promissor. A camada peritonial se regenera 5 a 7 dias após a cirurgia, independente da extensão do dano. A separação mecânica das superfícies das feridas durante este período pode prevenir a formação de aderências entre superfícies danificadas. Este conceito era defendido no final do século 19 por Vogel, através do emprego de solução de goma arábica, e no início do século 20 por Claypool e cols. com o uso de petrolato líquido ${ }^{68,69}$.

Recentemente foram desenvolvidos novos métodos na prevenção de aderências que incluem barreiras físicas (barreiras mecânicas sólidas, barreiras bioabsorvíveis em forma de gel ou filmes) e soluções macromoleculares.

A celulose oxidada regenerada Interceed $^{\circledR}$, Johnson \& Johnson) é um filme absorvível que leva à prevenção de aderências, sem o comprometimento da cicatrização, e pode ser utilizado durante cirurgia laparoscópica ou aberta, em qualquer localização intraperitoneal ${ }^{70}$. Apesar dos resultados inicialmente animadores, quando se observou eficácia duas vezes superior em pacientes submetidos à cirurgia 
ginecológica aberta ${ }^{71}$, o Interceed $^{\circledR}$ só deve ser utilizado após hemostasia rigorosa, uma vez que suas propriedades são inativadas em contato com o sangue, dificultando sua utilização em cirurgia geral.

A associação de ácido hialurônico e carboximetilcelulose (Seprafilm ${ }^{\circledR}$, Genzyme) resultou na redução na incidência, extensão, densidade e vascularização das aderências em pacientes submetidos à cirurgia colorretal em dois tempos, como após a retocolectomia total com anastomose íleo-anal com reservatório ileal e ileostomia de proteção ${ }^{72}$. Estudo multicêntrico em cirurgia colorretal com o uso de Seprafilm determinou a segurança da utilização deste produto; entretanto, observou-se maior incidência de deiscência de anastomose quando o filme foi utilizado nessas áreas específicas ${ }^{73}$. Outro estudo multicêntrico observou redução de $47 \%$ na incidência de aderências pós-operatórias em pacientes submetidos à ressecção intestinal; entretanto foram utilizados em média 4,4 unidades do filme, o que resultou em custo elevado do procedimento. ${ }^{74}$

A solução sintética de polietilenoglicol (Spraygel ${ }^{\circledR}$, Confluent Surgical) na forma de spray produz um filme de hidrogel quando aerossolizada. A barreira formada permanece aderida às alças por um período de sete dias, após os quais é excretada via renal. Os resultados obtidos com o uso do Spraygel ${ }^{\circledR}$ mostraram redução na incidência, gravidade e extensão de aderências pós-operatórias, mas a um custo ainda bastante elevado. ${ }^{75,76}$

Utilizada inicialmente em diálise peritoneal em concentrações mais elevadas, a solução de icodextrina a 4\% (Adept ${ }^{\circledR}$, Shire Pharmaceuticals) permanece por tempo prolongado na cavidade peritoneal, fazendo com que os órgãos intrabdominais flutuem na cavidade, afastados entre si, prevenindo a formação de aderências. Ao contrário dos agentes mencionados anteriormente, a utilização de $A d e p t^{\circledR}$ é tecnicamente fácil em laparoscopias e laparotomias e se mostrou bastante segura, com poucos efeitos deletérios. Além disso, não afeta a cicatrização das áreas anastomóticas. ${ }^{77,78}$

\section{IMPACTO DA VIA DE ACESSO NA PREVENÇÃO DE ADERÊNCIAS}

Apesar dos estudos experimentais apresentarem metodologia bastante variada entre si, muitos demonstram as vantagens da via laparoscópica sobre a convencional na prevenção e redução de aderências. Garrard e cols. ${ }^{79}$ avaliaram a ocorrência de aderências pós-operatórias em porcos e demonstraram que a colocação de tela de polipropileno por laparoscopia quando comparada à laparotomia ou mesmo à laparoscopia com incisão aumentada produziu menos aderência à tela no que se refere à extensão e ao grau de intensidade das aderências e à presença de vascularização.

Estudo realizado por Gamal e cols. ${ }^{80}$ em 60 cães submetidos à colecistectomia comparou as vias laparoscópica e convencional na formação de aderências. No segundo tempo operatório, os autores observaram que o grupo submetido à laparoscopia sem complicações operatórias não apresentou sinais de aderências, assim como o grupo no qual ocorreu perfuração acidental da vesícula biliar durante o procedimento laparoscópico. Por outro lado, aderências foram observadas no grupo submetido à colecistectomia pela via aberta e no grupo em que ocorreu sangramento ou lesão do leito hepático durante a laparoscopia. De forma semelhante, Tittel e cols. ${ }^{81}$ observaram menor formação de aderências em cães submetidos a ressecção do ceco por laparoscopia quando comparados à laparotomia.

Em estudo com porcos submetidos à nefrectomia transperitoneal, Moore e cols. ${ }^{82}$ compararam a formação de aderências pós-operatórias pós laparoscopia ou laparotomia, verificando incidência de $12,5 \%$ e $75 \%$, respectivamente. Enquanto no primeiro grupo as aderências estavam restritas à incisão dos portais, no segundo elas se estendiam por toda a incisão.

Em experiência com 52 ratos Sprague-Dawley comparando a extensão da formação de aderências após fundoplicatura por via laparoscópica ou aberta, Krahenbuhl e cols. ${ }^{83}$ observaram menor formação de aderências no grupo submetido à laparoscopia quando comparado à cirurgia convencional, de forma que no primeiro grupo as aderências eram predominantemente parietais e mais finas, enquanto que no segundo, eram predominantemente viscerais e mais espessas.

A formação de novas aderências em coelhos previamente submetidos a adesiólise pela via laparoscópica e pela laparotomia foi comparada por Tittel e cols. ${ }^{84}$. Os autores observaram que no grupo submetido a adesiólise pela laparotomia, a incidência 
de novas aderências foi de $100 \%$, enquanto que no grupo da laparoscopia esta foi de $75 \%$, sendo as aderências significativamente menos extensas. Os autores concluíram que a adesiólise laparoscópica leva a menor formação de novas aderências e, conseqüentemente, menor índice de complicações associadas.

Assim como os estudos experimentais já publicados, os estudos clínicos que comparam a incidência de aderências peritoniais quanto à via de acesso empregada carecem de uniformidade em sua metodologia, constatando-se diferença significativa quanto ao tipo de operação realizada, à técnica empregada pelo cirurgião e à forma de avaliação das aderências.

Lundorff e cols. ${ }^{85}$ avaliaram a incidência de aderências em pacientes com prenhez ectópica operadas pela via convencional ou por laparoscopia e observaram em segundo tempo cirúrgico que o tratamento laparoscópico levou à menor formação de aderências no lado operado quando comparado à laparotomia. Além disso, a avaliação do lado contralateral demonstrou menor intensidade de aderências no grupo submetido à laparoscopia, mas não se pôde associar significância estatística a este achado.

A ocorrência de aderências após coagulação de ovários policísticos foi o parâmetro avaliado por Taskin e cols. ${ }^{86}$ em estudo randomizado com 18 pacientes submetidas ao tratamento por laparoscopia convencional ou por minilaparoscopia com mínima exposição ao gás carbônico. Neste estudo, os autores observaram menor formação de aderências no grupo submetido ao tratamento pela minilaparoscopia quando comparado à técnica laparoscópica convencional, o que é, provavelmente, conseqüência da menor agressão do pneumoperitônio de baixo volume sobre a microcirculação peritonial e a estrutura celular.

Pacientes previamente submetidos à colecistectomia pela via laparoscópica $(n=18)$ ou pela via aberta $(\mathrm{n}=8)$ foram incluídos em estudo prospectivo não randomizado de Polymeneas e cols. ${ }^{87}$. Neste trabalho, os autores avaliaram a incidência de aderências peritoneais em uma segunda intervenção cirúrgica por patologias intrabdominais diversas. No grupo submetido à colecistectomia laparoscópica, não se observou aderências no lado operado ou relacionadas aos portais de acesso em $55 \%$ dos pacientes, enquanto que $16,6 \%$ dos pacientes apresentaram aderências mínimas no local operado. No grupo previamente submetido à colecistectomia pela via aberta, todos os pacientes apresentavam aderências densas no local operado ou na parede abdominal abaixo da incisão. Os autores concluíram que a laparoscopia esteve associada a menor incidência de aderências peritoniais pósoperatórias.

\section{AVALIAÇÃO DO CUSTO-EFETIVIDADE DE ESŢRATÉGIAS DE REDUÇÃO DE ADERENCIAS}

A indisponibilidade de estudos clínicos comparativos, associada à inexistência de dados quanto à ocorrência e à intensidade dos desfechos adversos das aderências peritoniais, tais como dor abdominal e pélvica, infertilidade e obstrução intestinal, torna a avaliação do impacto da laparoscopia sobre a incidência de aderências uma tarefa árdua.

A falta de estudos randomizados e controlados também prejudica a adoção de métodos de prevenção de aderências por muitos cirurgiões e administradores de sistemas de saúde, que relutam em introduzir essas técnicas com base em um número reduzido de estudos disponíveis.

Membros do grupo de estudo SCAR demonstraram que a realização de estudos randomizados e controlados com esse intuito envolveria um número exagerado de pacientes para atingir níveis significativos ${ }^{88}$. Supondo que o agente de prevenção da formação de aderências promovesse uma redução de $25 \%$ no número de internações relacionadas a aderências em um período de um ano, seria necessário incluir no estudo 5.586 a 7.786 pacientes para atingir significância estatística $(\mathrm{p}=0,05$ e $\mathrm{p}=0,01$, respectivamente). Ao considerar os pacientes perdidos durante o seguimento (10-15\%), seriam necessários de 6.539 a 8.148 pacientes e ao levarmos em conta os critérios de exclusão, esse número se elevaria para entre 13.000 e 16.000 pacientes.

A avaliação do custo da utilização de produtos para prevenção de aderências demonstrou que uma redução de $32,6 \%$ no número de internações por obstrução do intestino delgado no primeiro ano e de $16 \%$ após três anos seria necessária para justificar o gasto de 50 libras esterlinas por item utilizado. Entretanto, quando avaliados itens de 200 libras esterlinas, a redução de $100 \%$ no número de 
reinternações no primeiro ano não seria suficiente para cobrir os custos do produto, enquanto que em 3 anos, seria necessária uma redução de $64,1 \%$ no número de reinternações. Fazio e cols. ${ }^{74}$ demonstraram uma redução de $47 \%$ no número de internações por obstrução do intestino delgado após o uso de Seprafilm $^{\circledR}$. Baseado em estudo de Beck e cols. ${ }^{73}$, que estimou o custo da utilização de Seprafilm $^{\circledR}$ em torno de 350-450 libras esterlinas por paciente, conclui-se que uma redução de $100 \%$ no número de internações por oclusão do intestino delgado após 10 anos de seguimento não seria o suficiente para arcar com os custos do produto.

Em resumo, a realização de um estudo randomizado para verificar a efetividade de produtos para a redução de aderências é bastante discutível devido ao grande número de pacientes necessários e ao elevado custo associado aos produtos comercialmente disponíveis. Apesar de provavelmente representarem uma intervenção sem dano e, ao que tudo indica, associada a benefício, o uso clínico menos tímido das barreiras permanece "amarrado" como resultado de seu custo ainda elevado, sobretudo em nosso meio.

\section{CONSIDERAÇÕES FINAIS}

As aderências representam um problema já bastante conhecido após cirurgia abdominal e estão associadas a inúmeras complicações como infertilidade, dor abdominal e pélvica crônica e obstrução intestinal.

Acarretam elevado custo para o sistema de saúde. Aproximadamente 1 bilhão de dólares foram gastos nos Estados Unidos com internações para lise de aderências em $1998^{89}$.

A vídeo-cirurgia representa um avanço na prática cirúrgica na medida em que está associada a menor formação de aderências, o que pode ser atribuído às incisões menores, à menor introdução de corpos estranhos na cavidade, ao menor trauma resultante de afastadores e à menor manipulação de estruturas distantes do campo operatório. No entanto, é provável que quanto mais avançada for a operação por vídeo (por exemplo: a retocolectomia total videoassistida), será mais difícil verificarem-se os benefícios de menor formação de aderências pósoperatórias. $\mathrm{O}$ motivo seria a similaridade entre a extensão das áreas desperitonizadas na cirurgia laparoscópica colorretal avançada quando comparada à mesma operação realizada por via convencional. Estas áreas seriam similares exceto pelas relacionadas às incisões. Analogamente, complicações relacionadas à construção e fechamento de derivações de proteção mais comuns nas operações avançadas e sobre o reto estariam presentes em ambas as vias de acesso.

SUMMARY: Laparoscopic surgery seems to be associated with less adhesion formation and complications associated to surgical access when compared to laparotomy. Experimental and clinical evidence confirm this hypothesis but the impact of laparoscopy on adhesion formation and its complications remains undetermined. The present article aims at reviewing the evidence on this issue. Method: literature review. Results: Postoperative adhesions were evaluated at operation site and at surgical scars. Nevertheless, results on adhesion formation at sites distant from them are still unavailable. Adhesion formation was less common or reduced when laparoscopic access was compared to conventional surgery. Main adverse outcomes regarding adhesion formation are pelvic pain, infertility, and intestinal obstruction. There is little evidence of reduced incidence of these adverse outcomes after laparoscopic surgery when compared to conventional access and there may be none at all when major laparoscopic operations are considered. This finding may be due to a similar extent of dissection after conventional or advanced video operations with the exception of the adhesions related to the incisions. Conclusions: Laparoscopic surgery is associated to less adhesion formation but may not protect from adverse outcomes expected after abdominal operations. Adequate surgical technique and the use of commercially available adhesion barriers may be major determinants from adhesions formation and its consequences.

Key words: Adhesions, Laparoscopy, Intestinal Obstruction, Pelvic Pain, Infertility

\section{REFERÊNCIAS BIBLIOGRÁFICAS}

1. Menzies D, Ellis H. Intestinal obstruction from adhesionshow big is the problem? Ann Roy Coll Surg 1990, 72: 60-3.
2. Menzies D. Peritoneal adhesions. Incidence, cause, and prevention. Surg Annu 1992; 24: 27-45.

3. Soules MR, Dennis L, Bosarge A et al. The prevention of postoperative pelvic adhesions: an animal study comparing 
barrier methods with dextran 70. Am J Obstet Gynecol 1982; 143: 829-34.

4. Stovall TG, Elder RF, Ling FW. Predictors of pelvic adhesions. J Reprod Med 1989; 34: 345-8.

5. Van Der Krabben AA, Dijkstra FR, Nieuwenhuijzen M et al. Morbidity and mortality of inadvertent enterotomy during adhesiotomy. Br J Surg 2000; 87: 467-71.

6. Ellis H. The clinical significance of adhesions: focus on intestinal obstruction. Eur J Surg Suppl 1997; 577: 5-9.

7. Gutt CN, Oniu T, Schemmer Pet al. Fewer adhesions induced by laparoscopic surgery? Surg Endosc 2004; 18: 898-906.

8. Holmdahl L, Eriksson E, al-Jabreen $\mathrm{M}$ et al. Fibrinolysis in human peritoneum during operation. Surgery 1996; 119: 7015.

9. Holmdahl L, Eriksson E, Eriksson BI et al. Depression of peritoneal fibrinolysis during operation is a local response to trauma. Surgery 1998; 123: 539-44.

10. Vipond MN, Whawell SA, Thompson JN et al. Peritoneal fibrinolytic activity and intra-abdominal adhesions. Lancet 1990; 335: 1120-2.

11. Vipond MN, Whawell SA, Thompson JN et al. Effect of experimental peritonitis and ischaemia on peritoneal fibrinolyticactivity. Eur J Surg 1994; 160: 471-7.

12. van Goor H, Bom VJ, van der Meer J et al. Coagulation and fibrinolytic responses of human peritoneal fluid and plasma to bacterial peritonitis. Br J Surg 1996; 83: 1133-5.

13. van Goor H, de Graaf JS, Grond J et al. Fibrinolytic activity in the abdominal cavity of rats with faecal peritonitis. Br J Surg 1994; 81: 1046-9.

14. Ivarsson M-L, Holmdahl L, Eriksson E et al. Expression and kinetics of fibrinolytic components in plasma and peritoneum during abdominal surgery. Fibrinolysis Proteolysis 1998; 12 : 61-7.

15. Bakkum EA, Emeis JJ, Dalmeijer RA et al. Long-term analysis of peritoneal plasminogen activator activity and adhesion formation after surgical trauma in the rat model. Fertil Steril 1996; 66: 1018-22.

16. Scott-Coombes D, Whawell S, Vipond MN et al. Human intraperitoneal fibrinolytic response to elective surgery. Br J Surg 1995; 82: 414-7

17. Scott-Coombes DM, Whawell SA, Thompson JN. The operative peritoneal fibrinolytic response to abdominal operation. Eur J Surg 1995; 161: 395-9.

18. Milingos S, Kallipolitis G, Loutradis D et al. Adhesions: laparoscopic surgery versus laparotomy. Ann N Y Acad Sci 2000; 900: 272-85.

19. Vrijland WW, Jeekel J, Geldorp HJ et al. Abdominal adhesions: intestinal obstruction, pain, and infertility. Surg Endosc 2003; 17: 1017-22.

20. Moore RG, Partin AW, Adams JB et al. Adhesion formation after transperitoneal nephrectomy: laparoscopic v open approach. J Endourol 1995; 9: 277-80.
21. Ivarsson ML, Holmdahl L, Franzen G et al. Cost of bowel obstruction resulting from adhesions. Eur J Surg 1997; 163: 679-84.

22. Nagata $\mathrm{Y}$, Honjou K, Sonoda M et al. Peri-ovarian adhesions interfere with the diffusion of gonadotrophin into the folicular fluid. Hum Reprod 1998; 13: 2072-6.

23. Swank DJ, Van Erp WF, Van Repelaer Driel OJ et al. A prospective analysis of predictive factors on the results of laparoscopic adhesiolysis in patients with chronic abdominal pain. Surg Laparosc Endosc Percutan Tech 2003;13: 88-94.

24. De la Garza-Villasenor L. Etiology of intestinal occlusion. Rev Gastroenterol Mex 2001; 66: 193-6.

25. Tamijmarane A, Chandra S, Smile SR. Clinical aspects of adhesive intestinal obstruction. Trop Gastroenterol 2000; 21: 141-3.

26. Wysocki A, Krzywon J. Causes of intestinal obstruction. Przegl Lek 2001; 58: 507-8.

27. Ellis H. The magnitude of adhesion related problems. Ann Chir Gynaecol 1998; 87: 9-11.

28. Parker MC, Wilson MS, Menzies D et al. Colorectal surgery: the risk and burden of adhesion-related complications. Colorect Dis 2004; 6: 506-11.

29. Beck DE, Opelka FG, Bailey HR et al. Incidence of smallbowel obstruction and adhesiolysis after open colorectal and general surgery. Dis Colon Rectum 1999; 42: 241-8.

30. Richardson EH. Studies on peritoneal adhesions. With a contribution to the treatment of denuded bowel surfaces. Ann Surg 1911; 54: 758-97.

31. Boys F. The prophylaxis of peritoneal adhesions. A review of the literature. Surgery 1942; 11: 118-68.

32. Wiseman DM. Adhesion prevention: past the future. In Peritoneal Surgery, Dizerega GS (ed). Springer-Verlag: New York, 1999; 401-18.

33. Wright LT, Smith DH, Rothman M et al. Prevention of postoperative adhesions in rabbits with streptococcal metabolites. Proc Soc Exp Biol Med 1950; 75: 602-4.

34. James DCO, Ellis H, Hugh TB. The effect of streptokinase on experimental intraperitoneal adhesion formation. J Pathol Bacteriol 1965; 90: 279-87.

35. Lai HS, Chen Y, Chang KJ et al. Tissue plasminogen activator reduces intraperitoneal adhesion after intestinal resection in rats. J Formos Med Assoc 1998; 97: 323-7.

36. Orita H, Fukasawa M, Girgis W et al. Inhibition of postsurgical adhesions in a standardized rabbit model: intraperitoneal treatment with tissue plasminogen activator. Int J Fertil 1991; 36: $172-7$.

37. Montz FJ, Fowler JM, Wolff AJ et al. The ability of recombinant tissue plasminogen activator to inhibit post-radical pelvic surgery adhesions in the dog model. Am J Obstet Gynecol 1991; 165: 1539-42.

38. Vipond MN, Whawell SA, Scott-Coombes DM et al. Experimental adhesion prophylaxis with recombinant tissue plasminogen activator. Ann R Coll Surg Engl 1994; 76: 412-5. 
39. Buckenmaier CC III, Pusateri AE, Harris RA et al. Comparison of antiadhesive treatments using an objective rat model. Am Surg 1999; 65: 274-82.

40. Doody KJ, Dunn RC, Buttram VC Jr. Recombinant tissue plasminogen activator reduces adhesion formation in a rabbit uterine horn model. Fertil Steril 1989; 51: 509-12.

41. Menzies D, Ellis H. Intra-abdominal adhesions and their prevention by topical tissue plasminogen activator. J R Soc Med 1989; 82: 534-5.

42. Menzies D, Ellis $H$. The role of plasminogen activator in adhesion prevention. Surg Gynecol Obstet 1991; 172: 362-6.

43. Dunn RC, Mohler M. Effect of varying days of tissue plasminogen activator therapy on the prevention of postsurgical adhesions in a rabbit model. J Surg Res 1993; 54: 242-5.

44. Dunn RC, Steinleitner AJ, Lambert H. Synergistic effect of intraperitoneally administered calcium channel blockade and recombinant tissue plasminogen activator to prevent adhesion formation in an animal model. Am J Obstet Gynecol 1991; 164: 1327-30.

45. Evans DM, McAree K, Guyton DP, Hawkins N, Stakleff K. Dose dependency and wound healing aspects of the use of tissue plasminogen activator in the prevention of intra-abdominal adhesions. Am J Surg 1993; 165: 229-32.

46. Lehman EP, Boys F. The prevention of peritoneal adhesions with heparin. An experimental study. Ann Surg 1940; 111: 427-35.

47. Fukasawa M, Girgis W, diZerega GS. Inhibition of postsurgical adhesions in a standardized rabbit model. II. Intraperitoneal treatment with heparin. Int J Fertil 1991; 36: 296-301.

48. Lu HJ, Chang Y, Sung HW et al. Heparinization on pericardial substitutes can reduce adhesion and epicardial inflammation in the dog. J Thorac Cardiovasc Surg 1998; 115: 1111 20.

49. Noishiki Y, Miyata T. Antiadhesive collagen membrane with heparin slow release. J Bioact Compat Polym 1987; 2: 325 33.

50. Chalkiadakis G, Kostakis A, Karayannacos PE et al. The effect of heparin upon fibrinopurulent peritonitis in rats. Surg Gynecol Obstet 1983; 157: 257-60.

51. Turkcapar AG, Ozarslan C, Erdem E et al. The effectiveness of low molecular weight heparin on adhesion formation in experimental rat model. Int Surg 1995; 80: 92-4.

52. Gupta S, Jain PK. Low-dose heparin in experimental peritonitis. Eur Surg Res 1985; 17: 167-72.

53. O'Leary JP, Malik FS, Donahoe RR et al. The effects of a minidose of heparin on peritonitis in rats. Surg Gynecol Obstet 1979; 148: 571-5.

54. Tayyar M, Basbug M. The effects of intraperitoneal piroxicam and low molecular weight heparin in prevention of adhesion reformation in rat uterine horn. Res Exp Med 1999; 198: 269 75.
55. Vela AR, Littleton JC, O'Leary JP. The effects of minidose heparin and low molecular weight heparin on peritonitis in the rat. Am Surg 1999; 65: 473-7.

56. Siegler AM, Kontopoulos V, Wang CF. Prevention of postoperative adhesions in rabbits with ibuprofen, a nonsteroidal anti-inflammatory agent. Fertil Steril 1980; 34: 46-9.

57. Swolin K. Die einwirkung von grossen, intraperitonealen dosen glukokorticoid auf die bildung von postoperativen adhäsionen. Klinische studien mit hilfe des laparoskopes an operierten extrauteringraviditäten. Acta Obstet Gynecol Scand 1967; 46: 204-18.

58. Jansen RP. Failure of intraperitoneal adjuncts to improve the outcome of pelvic operations in young women. Am J Obstet Gynecol 1985; 153: 363-71.

59. Larsson B. Prevention of postoperative formation, reformation of pelvic adhesions. In Peritoneal Adhesions, Treutner KH, Schumpelick V (eds). Springer: Berlin, 1997; 331-4.

60. Nishimura K, Nakamura RM, diZerega GS. Biochemical evaluation of postsurgical wound repair: prevention of intraperitoneal adhesion formation with ibuprofen. J Surg Res 1983; 34: 219-26.

61. Wiseman DM, Huang WJ, Johns DB et al. Time-dependent effect of tolmetin sodium in a rabbit uterine adhesion model. J Invest Surg 1994; 7: 527-32.

62. Celebioglu B, Eslambouli NR, Olcay E et al. The effect of tenoxicam on intraperitoneal adhesions and prostaglandin E2 levels in mice. Anesth Analg 1999; 88: 939-42.

63. LeGrand EK, Rodgers KE, Girgis W et al. Comparative efficacy of nonsteroidal anti-inflammatory drugs and anti-thromboxane agents in a rabbit adhesion-prevention model. J Invest Surg 1995; 8: 187-94.

64. LeGrand EK, Rodgers KE, Girgis W et al. Efficacy of tolmetin sodium for adhesion prevention in rabbit and rat models. $\mathrm{J}$ Surg Res 1994; 56: 67-71

65. Muzii L, Marana R, Brunetti L et al. Postoperative adhesion prevention with low-dose aspirin: effect through the selective inhibition of thromboxane production. Hum Reprod 1998; 13: 1486-9.

66. Nagler A, Rivkind AI, Raphael J et al. Halofuginone-an inhibitor of collagen type I synthesis-prevents postoperative formation of abdominal adhesions. Ann Surg 1998; 227: 57582.

67. Nagler A, Genina O, Lavelin I et al. Halofuginone, an inhibitor of collagen type I synthesis, prevents postoperative adhesion formation in the rat uterine horn model. Am J Obstet Gynecol 1999; 180: 558-63.

68. Vogel K. Clinical and experimental study of peritoneal adhesion occurring after laparotomy. Deutsche Zeit Chir 1898; ixiii: 296 (citação em Ann Surg 1902 36: 961-2).

69. Claypool JR, Vance BM, Robertson PR et al. A study in the prevention of adhesions. JAMA 1910; 55: 312-3. 
70. Interceed (TC7) Adhesion Barrier Study Group. Prevention of postsurgical adhesions by INTERCEED (TC7), an absorbable adhesion barrier: a prospective randomized multicenter clinical study. Fertil Steril 1989; 51: 933-8.

71. Wiseman DM, Trout JR, Franklin RR et al. Metaanalysis of the safety and efficacy of an adhesion barrier (Interceed TC7) in laparotomy. J Reprod Med 1999; 44: 325-31.

72. Becker JM, Dayton MT, Fazio VW et al. Prevention of postoperative abdominal adhesions by a sodium hyaluronate-based bioresorbable membrane: a prospective, randomized, doubleblind multicenter study. J Am Coll Surg 1996; 183: 297-306.

73. Beck DE, Cohen Z, Fleshman JW et al. A prospective, randomized, multicenter, controlled study of the safety of Seprafilm adhesion barrier in abdominopelvic surgery of the intestine. Dis Colon Rectum 2003; 46: 1310- 9.

74. Fazio V. An Initial Report on the Effectiveness of Seprafilm ${ }^{\circledR}$ in the Reduction of Occurrence of First Bowel Obstruction. American Society of Colon and Rectal Surgeons Annual Meeting 2004.

75. Mettler L, Audebert A, Lehmann W, Schive K, Jacobs VR. Prospective clinical trial of SprayGel as a barrier to adhesion formation: an interim analysis. J Am Assoc Gynecol Laparosc 2003; 10: 339-44.

76. Johns DA, Ferland R, Dunn R. Initial feasibility study of a sprayable hydrogel adhesion barrier system in patients undergoing laparoscopic ovarian surgery. J Am Assoc Gynecol Laparosc 2003; 10: 334-8.

77. Hosie K, Gilbert JA, Kerr D, Brown CB, Peers EM. Fluid dynamics in man of an intraperitoneal drug delivery solution: 4\% icodextrin. Drug Deliv 2001; 8: 9-12.

78. Verco SJ, Peers EM, Brown CB, Rodgers KE, Roda N, diZerega G. Development of a novel glucose polymer solution (icodextrin) for adhesion prevention: pre-clinical studies. Hum Reprod 2000; 15: 1764-72.

79. Garrard CL, Clements RH, Nanney Let al. Adhesion formation is reduced after laparoscopic surgery. Surg Endosc 1999;13:10-3.

80. Gamal EM, Metzger P, Miko I et al. The judgement of adhesion formation following laparoscopic and conventional cholecystectomy in an animal model. Acta Chir Hung 1999; 38:169-72.
81. Tittel A, Schippers E, Anurov M et al. Minor abdominal trauma by laparoscopic surgery? Comparison of adhesion formation and intestinal motility after laparoscopic and conventional operations in the dog. Zentralbl Chir 1996; 121: 329-34.

82. Moore RG, Partin AW, Adams JB et al. Adhesion formation after transperitoneal nephrectomy: laparoscopic v open approach. J Endourol 1995; 9: 277-80.

83. Krahenbuhl L, Schafer M, Kuzinkovas V et al. Experimental study of adhesion formation in open and laparoscopic fundoplication. Br J Surg 1998; 85: 826-30.

84. Tittel A, Treutner KH, Titkova $\mathrm{S}$ et al. New adhesion formation after laparoscopic and conventional adhesiolysis: a comparative study in the rabbit. Surg Endosc 2001; 15: 44-6.

85. Lundorff $\mathrm{P}$, Hahlin M, Kallfelt B et al. Adhesion formation after laparoscopic surgery in tubal pregnancy: a randomized trial versus laparotomy. Fertil Steril 1991; 55: 911-5.

86. Taskin O, Sadik S, Onoglu A et al. Adhesion formation after microlaparoscopic and laparoscopic ovarian coagulation for polycystic ovary disease. J Am Assoc Gynecol Laparosc 1999; 6: 159-63.

87. Polymeneas G, Theodosopoulos T, Stamatiadis A et al. A comparative study of postoperative adhesion formation after laparoscopic vs open cholecystectomy. Surg Endosc 2001; 15: 41-3.

88. Wilson MS, Menzies D, Knight A, Crowe AM. Demonstrating the clinical and cost effectiveness of adhesion reduction strategies. Colorectal Dis 2002; 4: 355-60.

89. Ray NF, Larsen JW Jr, Stillman RJ et al. Economic impact of hospitalizations for lower abdominal adhesiolysis in the United States in 1988. Surg Gynaecol Obstet 1993; 176: 271-6.

Endereço para correspondência:

SÉRGIO EDUARDO ALONSO ARAUJO

Rua Cristiano Viana, 450 apt. 62

05411-000, São Paulo (SP)

E-mail: sergioaraujo@colorretal.com.br 